\title{
Effects of particle size and concentration on the pulsation characteristics of transformer oil
}

\author{
$\mathrm{C} \mathrm{Bin}^{1^{*}}$ and $\mathrm{L} \mathrm{Ge} \mathrm{Ge}^{2}$ \\ ${ }^{1}$ School of Mechanical \& Electrical, Hebei Key Laboratory of Safety Monitoring of Mining Equipment, North China Institute of Science and \\ Technology, Hebei, 065201, China. \\ ${ }^{2}$ Heibei Key Laboratory of Hazardous Chemicals Safety and Control Technology, School of Chemical and Environmental Engineering, North \\ China Institute of Science and Technology, Hebei, 065201, China.
}

\begin{abstract}
The presence of particulate matter in oil has a great impact on the pulsation characteristics of oil. The effects of particle size and concentration on the pulsation characteristics of oil were studied to ensure the safe operation of oil equipment. According to the ISO 4406 standard for oil contamination, oil samples containing $\mathrm{Cu}, \mathrm{Fe}$, and $\mathrm{SiO}_{2}$ with particle sizes of 5, 15,25 , and $50 \mu \mathrm{m}$ and concentrations of $6.58,4.57,3.00,0.971$, $0.648 \mathrm{ppm}$ were configured. Oil flow with different particle sizes and concentrations was tested in a square tube using PIV measurement technology. The instantaneous velocity vector data of the flow field were acquired in oil with different sizes and concentrations of the particles, and the distributions of the pulsation intensity of oil along the streamwise and normal directions were analysed. The time-frequency curves of the transient velocity and average velocity were discussed. The results showed that the distribution of the streamwise pulsation intensity of oil changes more gently along the normal direction in the centre region and changes more steeply in the near-wall area. With increasing particle concentration, the streamwise pulsation intensity of oil decreases gradually. The distribution of the normal pulsation intensity of oil along the normal direction is rough ' $w$ '-shaped under different particle diameters. With increasing particle concentration, the main frequency trend of the oil speed along with the streamwise and normal directions decreases, and the streamwise average speed of the oil increases. The change in the normal average speed of oil decreases when the particle concentration is under $3.00 \mathrm{ppm}$. The normal velocity of oil increases when the particle concentration is over $3.00 \mathrm{ppm}$.
\end{abstract}

Keywords: Oil flow, particle concentration, particle size, PIV measurement, pulsation characteristics.

\section{INTRODUCTION}

The power transformer is the core equipment of power transmission and conversion that plays a significant role in ensuring the safe operation of the power grid. However, the transformer pulsates due to the influence of various factors, such as core loosening and winding deformation, which will significantly reduce the ability of the transformer to withstand short-circuit impact and then cause power system faults. Therefore, monitoring the pulsation of transformers and evaluating the state of core and winding is of great significance for the stable operation of transformers. The vibration of a transformer is caused by winding deformation due to the electromagnetic force on the winding through the transformer oil to the tank wall or loose core due to magnetostriction phenomenon of silicon steel sheet by the same transformer oil to the tank wall, so the transformer pulse monitoring can be done by detecting the transformer oil pulsation (Bagheri, 2018). In the industry, various methods are usually used to detect the pulsation of transformer oil to obtain the fault information of a transformer indirectly because the pulsation of transformer oil is not only related to 
the function of the transformer switching switch but also plays a vital role in the pulsation of oil due to the characteristics of the oil, particle size and concentration in the oil (Zeng, 2018).

As an arc extinguishing medium, transformer oil is bound to produce carbon particles, polar impurities, and charging colloids and other ageing products. Moreover, with the increase of switching switch or selector switch connection conversion times, there must be mechanical friction and wear in the oil. Mechanical wear debris, mainly copper, iron, and other metal particles directly adulterate the transformer oil. These particles are very fine, and some of them can float in the oil for a long time without settling. Moreover, iron dust, even by the transformer winding adsorption, seriously affects the insulation performance of the transformer (Borges, 2015). Therefore, it is necessary to carry out studies to understand the influence of the size and concentration of particles in the transformer oil on the pulsation characteristics of oil for the safe operation of the transformer.

The effect of particle size and concentration on the pulsation characteristics of oil belongs to a sparse solidliquid two-phase flow field, on which many scholars have carried out theoretical simulation and experimental research (Abiev \& Galushko, 2013; Eschmann et al., 2015; Wang et al., 2016; Yuan et al., 2016). These results have a specific reference value to investigate the influence of particle size and concentration in oil with higher viscosity on the pulsation characteristics of the oil.

Papadopoulos et al. (2016) conducted a direct numerical simulation of sinusoidal pulsation turbulence in a straight tube with a low-volume Reynolds number and a high-frequency, indicating the evolution of the average velocity and fluid pulsation over time. Tian et al. (2016) obtained the variation trend of vibration displacement and the velocity of a pipeline system by establishing an analysis method of pressure pulsation in the channel and the coupling interaction of tube flow. Yan et al. (2012) studied the interaction between quasiperiodic large-scale vortex structures and flow pulsation in a rectangular channel. The conclusions indicated that the pulsation of the vortex structure and the flow velocity pulsation could be enhanced by an adjacent velocity interface. Zhao et al. (2016) studied the unsteady flow field in a channel using direct numerical simulation and found that the contributions of the lowfrequency vortices to the flow direction and normal pulsation velocity increase with increasing normal height; the pulsation of the spanwise velocity at the nearwall surface exhibited a large pulsation strength in the logarithmic layer and the viscous bottom layer. Ghadi et al. (2016) conducted jet oscillating flow experiments and showed that pulsating flow forms a coherent periodic structure. The pulsation frequency has a significant influence on the formation, size, and dynamics of a vortex structure. Hsu et al. (2014) measured a jet field using high-speed particle image velocimetry (PIV). They found that with the change in the jet exit velocity, the jet produced vibration within a pulsating period and induced a periodic wavy flow structure in the downstream region. Yang et al. (2013) believed that the change in frequency of wind-sand flow was at least greater than $100 \mathrm{~Hz}$, and the effect of atmospheric turbulent pulsation on sediment concentration was related to the sand grain size; the smaller the particle size was, the greater the effect of wind speed fluctuation.

Two-phase flow in pipelines has been studied for the generation and development of flow field pulsation and the interaction of vortex structures in flow fields at home and abroad. However, due to the random nature of oil movement and the complexity of its interaction with particles, the understanding of the pulsation characteristics of oil with different particle sizes and concentrations remains in the qualitative or semiquantitative stage. Moreover, there is little literature that analyses the pulsation characteristics of oil with higher viscous fluids. Therefore, it is necessary to investigate the pulsation characteristics of oil in the flow direction and for the normal pulsation strength, as well as the instantaneous velocity and the average velocity based on the vector field data measured by the PIV of the oil-containing particles in pipelines. Additionally, understanding the pulsation movement of oil at different concentrations and particle sizes reveals the interaction between particles and oil and lays a foundation for understanding the physical essence of the formation and development of oil pollution. This enables effective detection and diagnosis of transformer pulsation, early detection and elimination of hidden faults, avoiding unexpected accidents, and is vital for the safe operation of the transformer.

\section{METHODOLOGY}

The PIV experimental equipment for oils containing different particle sizes and particle concentrations are shown in Figure 1. It is mainly divided into two parts: experimental pipeline and PIV testing device. The coordinate origin is the vertex at the lower left corner of the experimental pipeline, $x$-direction is the flow direction of oil, y-direction is the spanwise direction, 
and $\mathrm{z}$-direction is the normal direction of oil. The experimental pipeline is a square tube with a total length of $500 \mathrm{~mm}$ and a cross-section of $40 \times 40 \mathrm{~mm}$, the oil inlet section of the pipeline is the development section $200 \mathrm{~mm}$ long and the middle is the test section $100 \mathrm{~mm}$ long, the bottom and back wall of the test section is made of a steel plate, the front wall and top surface are made of glass and can be disassembled for PIV laser incidence and testing. 2DPIV system from TSI (USA) is used for the PIV test device,

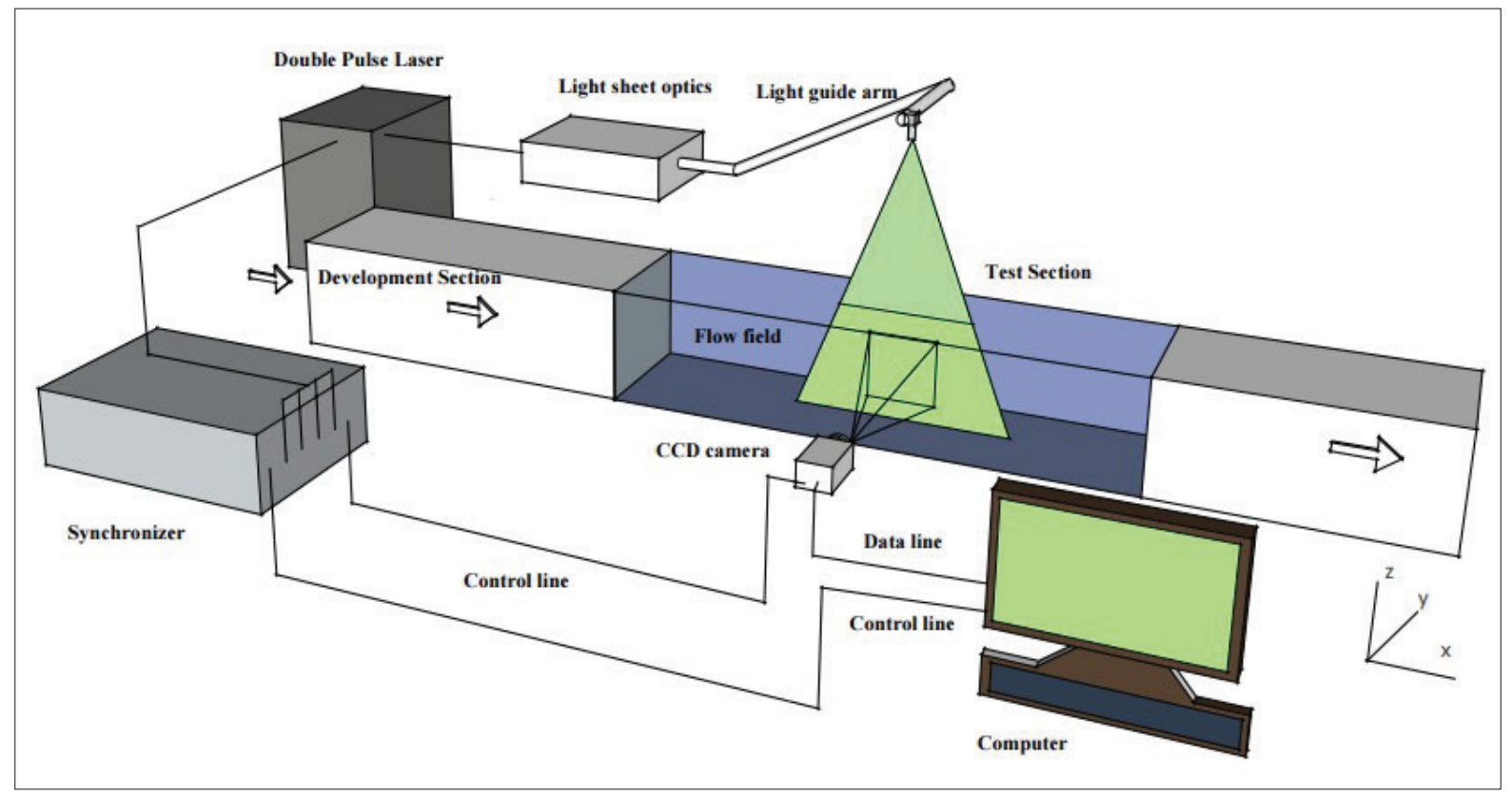

Figure 1: PIV experimental equipment for oil liquid

The 2DPIV system consists of a double yttrium aluminum garnet laser $(200 \mathrm{~mJ}$, pulse frequency $15 \mathrm{~Hz}$, laser wavelength $532 \mathrm{~nm}$ ), light guide arm, sheet light source lens (focal length from 30-3000 mm, two sets of cylindrical lenses $15 \mathrm{~mm}$ F.L. and $-25 \mathrm{~mm}$ F.L., and divergence angles $25^{\circ}$ and $14^{\circ}$, respectively), a Nikon CCD camera (15 frames per sec, $50 \mathrm{~mm} / \mathrm{F} 1.8$ lens, $2 \mathrm{~K} \times 2 \mathrm{~K}$ pixel resolution) and TSI Insight4G image acquisition, analysis and display software platform. The coordinate frame of the camera is three-dimensional and is automatically controlled by a microcomputer with a movement accuracy of $0.01 \mathrm{~mm}$. The laser light emitted from the laser passes through the light guide arm and the light source lens to form a 1-mm thick sheet light, which is irradiated from the normal direction (z-direction) of the pipe test section. Then, oil image information is obtained by the camera positioned in the direction of the pipe (y-direction). In addition, the synchronization controller (time resolution of $0.25 \mathrm{~ns}$ ) is set to trigger the laser and the camera synchronously.
25\# transformer oil was used as the test fluid (colourless and transparent for the PIV test). First, the oil was treated by a vacuum filter to obtain an initial oil sample. Then, $\mathrm{Cu}, \mathrm{Fe}$, and $\mathrm{SiO}_{2}$ powders (Beijing Focus Yingchuang Technology Co., Ltd., China) of different particle sizes were selected as particulate matter to simulate metallic and non-metallic contaminants in transformer oil. According to the ISO 4406 standard for oil contamination, the particulate matter in oil was divided into several size segments of $5,15,25$, and $50 \mu \mathrm{m}$ according to different contamination levels. The concentration of particulate matter in the measured oil sample was converted into the corresponding volume fraction of particulate matter in oil according to the test results of different contamination levels. The volume fraction of the particulate matter in oil was obtained as $6.58,4.57,3.00,0.971,0.648$ ppm in several volume concentrations of oil samples. In this method, $0.02 \mathrm{~g}$ of $\mathrm{Cu}, \mathrm{Fe}$, and $\mathrm{SiO}_{2}$ powder were mixed with $1 \mathrm{~L} 25 \#$ transformer oil and oscillated evenly in an ultrasonic oscillator for 8 hours (temperature kept at 
$\left.30-60{ }^{\circ} \mathrm{C}\right)$. Then, the mixed solution was separated using a filter paper having different pore diameters to obtain oil samples of medium diameter of $5,15,25$, and $50 \mu \mathrm{m}$ and a concentration of $14.40 \mathrm{ppm}$. Next, the initial oil sample was added to the oil sample at a certain volume ratio, and ultrasonic vibration was evenly distributed over 8 hours (temperature kept at $30-60{ }^{\circ} \mathrm{C}$ ). Oil samples containing particles having a gradient concentration of $6.58,4.57$, $3.00,0.971$, and $0.648 \mathrm{ppm}$ of different medium-diameter particles were eventually obtained.

Since the pulsation amplitude of transformer oil in the operational process is small at about $100 \mu \mathrm{m}$, and lower pulsation frequency of about $100 \mathrm{~Hz}$, the oil in the transformer is in laminar flow. So, the initial velocity of the oil inlet is set as $\mathrm{V}_{0}=0.0362 \mathrm{~m} / \mathrm{s}$. As the dynamic viscosity $\mu$ of transformer oil is $10.58 \mathrm{mPa}$.s, density $\rho$ is $895 \mathrm{~kg} / \mathrm{m}^{3}$, equivalent diameter $\mathrm{d}$ of the experimental pipe section is $0.0452 \mathrm{~m}$, corresponding Reynolds number Re $=\rho d \mathrm{~V}_{0} / \mu=138.3$, and the oil in the pipeline is in laminar flow. Since the temperature did not change much during an experiment, the influence of temperature was not considered. The oil samples to be tested were thoroughly mixed and fed into the test pipeline through a peristaltic pump. As the particle size is up to $50 \mu \mathrm{m}$, the dynamic viscosity of transformer oil $10.58 \mathrm{mPa} . \mathrm{s}$ is larger, thus after flowing through the pipeline development section of $200 \mathrm{~mm}$ long, a uniform liquid-solid two-phase suspension flow was formed in the test section, and then entered the oil tank through the tail section to form a circulation. After several experimental tests, the system was run for 30 minutes before the PIV test, ensuring that the oil and particles were fully and uniformly mixed during the PIV measurement period.

As the particle size in oil is less than $100 \mu \mathrm{m}$, the particles follow good behaviour and uniform distribution of light scattering, so no tracer particles were needed to be added during the test. The acquired image adopts the algorithms of multigrid iterative mutual correlation and multiadaptive deformation window. To obtain the original velocity vector, the image query area adopts pipeline horizontal and vertical initial query windows of $72 \times 64$ pixels, a final query window of $64 \times 48$ pixels and $50 \%$ overlap to eliminate the impact of the query window boundary. The method of processing the error vector is to use a local average verification method, and this vector is replaced by the mean in the adjacent $5 \times 5$-pixel range. The measurement position is $300 \mathrm{~mm}$ from the inlet of the pipeline; the measurement area is (flow direction $\times$ normal direction) $85.86 \times 22.20 \mathrm{~mm}$, while the centre of the area is (flow direction $\times$ normal direction) $300 \times 30 \mathrm{~mm}$. In addition, 200 image frames were captured in each test period.

\section{RESULTS AND DISCUSSION}

The instantaneous velocity vector field data were averaged over time, and the mean square of the flow velocity and normal pulsation velocity were obtained. The flow pulsation intensity and normal pulsation intensity were as follows:

$\sigma_{u}=\sqrt{\frac{1}{\mathrm{C}} \sum_{x=1}^{\mathrm{C}}\left[u(x, y, t)-\frac{1}{\mathrm{~N}} \sum_{\mathrm{i}=1}^{\mathrm{N}} u(x, y, t)\right]^{2}}$,

$\sigma_{v}=\sqrt{\frac{1}{\mathrm{C}} \sum_{x=1}^{\mathrm{c}}\left[v(x, y, t)-\frac{1}{\mathrm{~N}} \sum_{\mathrm{i}=1}^{\mathrm{N}} v(x, y, t)\right]^{2}}$

In the formulas, $\sigma_{u}$ and $\sigma_{v}$ indicate the fluctuation intensity of the flow and normal pulsation intensities, respectively; $u(x, y, t)$ and $u(x, y, t)$ represent the flow instantaneous velocity and normal instantaneous velocity at a certain position (x.y) at a certain time t, respectively, that PIV acquires in the flow field; $\mathrm{N}$ is the period of PIV acquisition, and $\mathrm{C}$ is the number of acquisitions in the PIV field of view.

\section{Distribution of flow pulsation intensity along the normal direction}

When the particle size in the oil is $5,15,25$, and $50 \mu \mathrm{m}$ while the particle concentration is $0.65,0.97,3.00,6.58$, and $14.40 \mathrm{ppm}$, the distribution curves of the flow pulsation intensity along the normal direction are shown in Figure 2. The horizontal axes of the graphs show the ratio of the flow pulsation intensity to the fluid inlet velocity $\mathrm{V}_{0}$, and the vertical coordinate represents the normal dimensionless pipe length.

Figure 2(a) shows the distribution of the flow pulsation intensity of the oil along the normal direction with different particle concentrations for a particle size of $5 \mu \mathrm{m}$. The figure shows that the flow pulsation intensity of the PIV acquisition oil field is relatively slow to change along the normal direction in the central region due to the presence of particulate matter, while the change is steep in the near-wall area, and this trend becomes increasingly obvious as the particle concentration increases. On the other hand, when the particle concentration is lower, the flow pulsation intensity of the oil field is larger in the near-wall area and gradually decreases toward the centre of the pipe; however, this trend becomes less obvious as the particle concentration increases. At the same concentration near the $y / d=0.25$ normal position, the flow pulsation intensity of the oil appears as a maximum. 
Overall, with increasing particle concentration, the flow pulsation intensity of the oil successively decreases. If the particle concentration is too large (such as 6.58 and $14.40 \mathrm{ppm}$ ), the difference in flow pulsation intensity is smaller. The reason is that at a constant concentration near the $y / d=0.25$ normal position is the normal centre of the oil laminar flow. The flow intensity of the oil appears as a parabolic flow pattern, thus having a maximum value at this position. The particles in oil in the near-wall area increase the flow velocity of the oil. To maintain mass conservation, the oil flow velocity in the central region decreases. Additionally, coupled with the blocking action of the oil particles, the flow pulsation

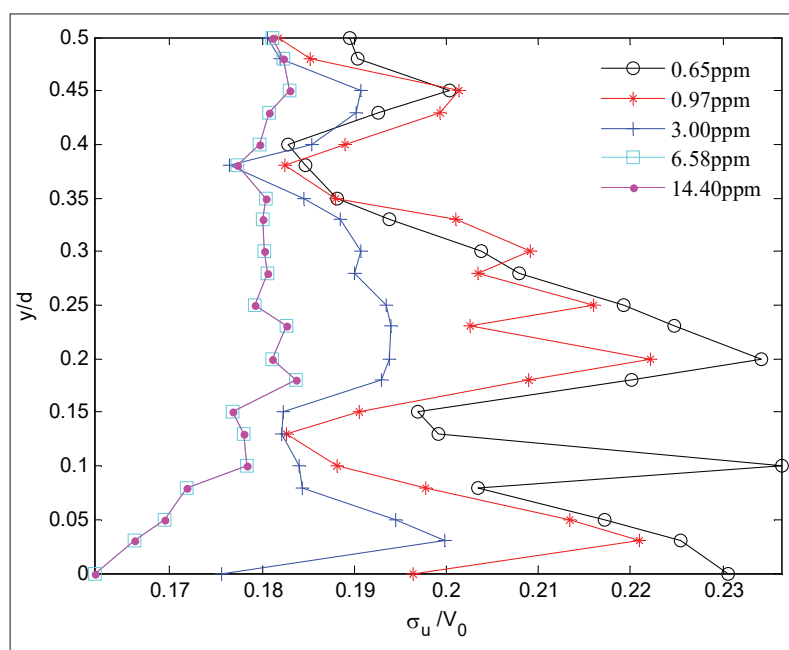

(a) $5 \mu \mathrm{m}$

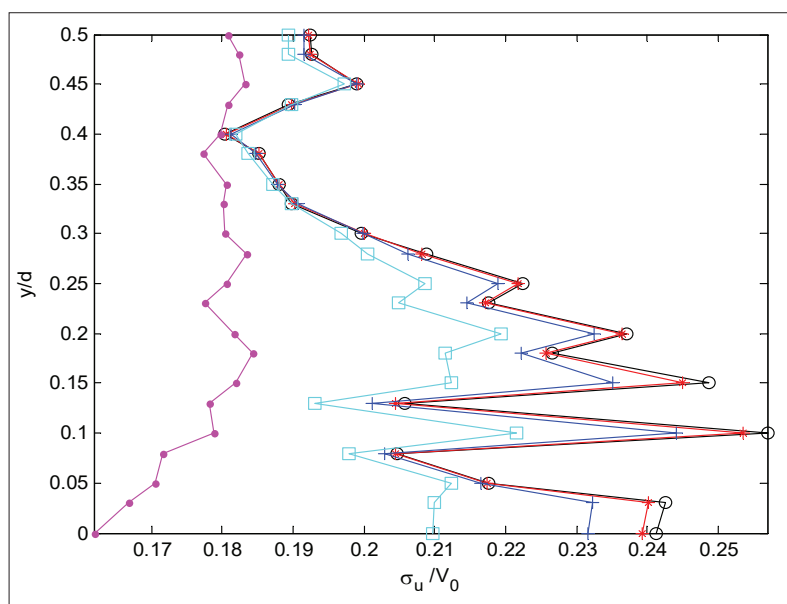

(c) $25 \mu \mathrm{m}$ intensity of the oil is steeply distributed in the near-wall region along the normal direction, while this intensity is relatively gentle in the centre area (Ling \& Zhong, 1999). In addition, with increasing particle concentration in oil, the effect of near-wall particles on oil flow velocity growth is attenuated due to decreasing viscous shear force, which weakens the tendency of oil flow pulsation intensity growth in the near-wall area. Therefore, as the particle concentration reaches $3.00 \mathrm{ppm}$ or more, the tendency of the flow pulsation intensity distribution along the normal direction becomes slower. This finding also indicates that the flow pulsation of the flow field in the pipeline is greatly affected by the wall surface.

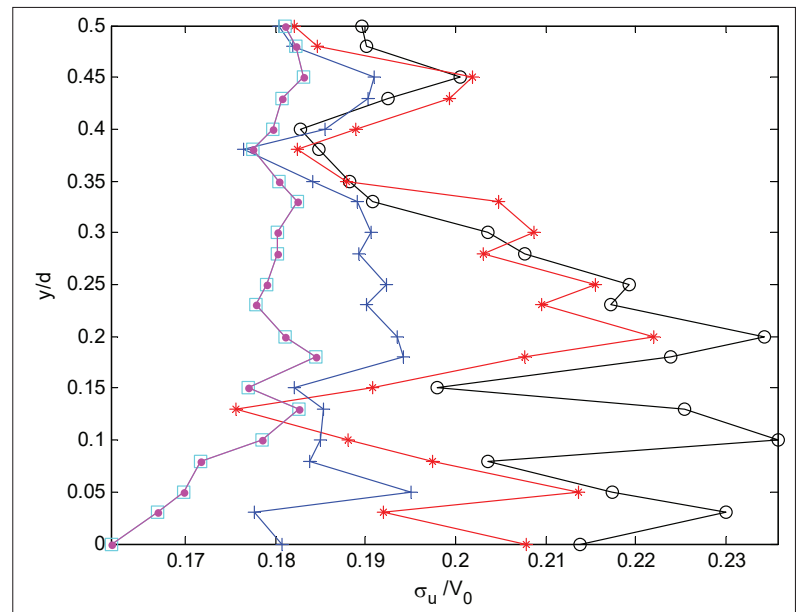

(b) $15 \mu \mathrm{m}$

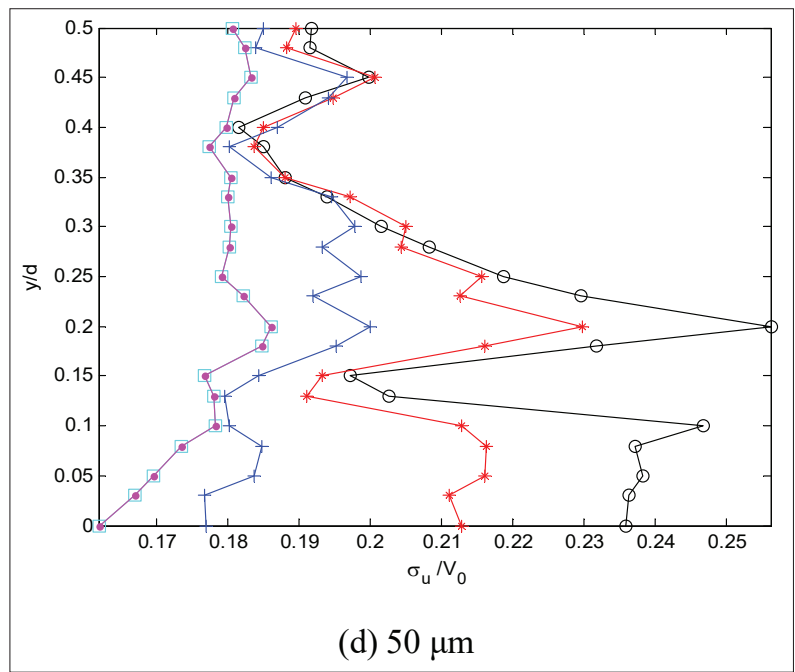

Figure 2: The distribution of streamwise mean intensity along the normal direction 
According to the effect of the $15 \mu \mathrm{m}$ particle size on the flow pulsation intensity of oil as shown in Figure 2(b), the effect of different particle concentrations on the intensity distribution along the normal direction is consistent with that of the $5 \mu \mathrm{m}$ particle size shown in Figure 2(a). The difference is that in the case of low particle concentration, the maximum value of the flow pulsation intensity is larger for the $15 \mu \mathrm{m}$ particle size. For example, the maximum value of the flow pulsation intensity for the $5 \mu \mathrm{m}$ particle size under $0.65 \mathrm{ppm}$ particle concentration is 0.233 , while the maximum flow pulsation intensity for the $15 \mu \mathrm{m}$ particle size is 0.236 . The reason is that the larger the particle size is, the greater the viscous shearing force of the oil, so the particles in the near-wall region increase the role of oil flow velocity growth.

Figure 2(c) shows the effect of different particle concentrations on the flow pulsation intensity of the oil when the particle size is $25 \mu \mathrm{m}$. The effect of the $25 \mu \mathrm{m}$ particle size on the flow pulsation intensity of the oil is similar to the trends shown in Figure 2(a) and (b). However, for the case of the particle size of $25 \mu \mathrm{m}$, the flow pulsation intensity is larger in the near-wall region and has a value of 0.256 , and then, the $5 \mu \mathrm{m}$ and $15 \mu \mathrm{m}$ amplitudes are increased by 9.95 and $8.56 \%$, respectively. In addition, when the particle concentration is $6.58 \mathrm{ppm}$, the flow pulsation intensity of oil is larger. This finding indicates that the particle size in the oil has less attenuation effect on the viscous shear force of oil when the particle size is $25 \mu \mathrm{m}$. However, the effect of the particles in the near-wall area on the oil growth rate is enhanced when the particle size exceeds $25 \mu \mathrm{m}$, due to the increase in particle size to enhance its gravitational sedimentation, and thus under the combined effect of viscous shear and gravity when the particle size is $50 \mu \mathrm{m}$ as shown in Figure 2(d), the amplitude of the flow pulsation intensity under each particle concentration decreases greatly, except for the particle concentration of $0.65 \mathrm{ppm}$. Moreover, when the particle concentration is $6.58 \mathrm{ppm}$, the flow pulsation intensity is the same as that for $14.40 \mathrm{ppm}$.

The results in Figure 2 show that the flow pulsation intensity decreases with increasing particle concentration, and the difference decreases gradually for oil with different particle concentrations. The intensity of flow pulsation increases simultaneously with the increase of particle size.

\section{Normal pulsation intensity distribution along the normal direction}

Figure 3 shows the distribution curves of the normal pulsation intensity along the normal direction when the particle sizes are is $5,15,25$, and $50 \mu \mathrm{m}$ and the particle concentrations are $0.65,0.97,3.00,6.58$, and $14.40 \mathrm{ppm}$. The horizontal axes in the figures show the ratio of the normal pulsation intensity to the oil inlet velocity $\mathrm{V}_{0}$, and the vertical coordinate represents the dimensionless length of the pipeline.

Figure 3 shows that the distribution of the normal pulsation intensity of oil with different particle sizes along the normal direction is roughly of the shape of a ' $W$ '. The amplitude of the normal pulsation intensity in the central region and the near-wall region is largely within the field of view tested by PIV. Moreover, the particle concentration has a non-unidirectional effect on the distribution of the normal pulsation intensity. The normal pulsation intensity is also affected by the pipeline wall, but the distribution of the normal pulsation intensity shows a parabolic shape due to the action of the central mainstream.

Figure 3(a) shows the effect of different particle concentrations on the distribution of the normal pulsation intensity when the particle size is $5 \mu \mathrm{m}$. The particle concentration (6.58 and $14.40 \mathrm{ppm}$ ) is larger, and the variation in the normal pulsation intensity of the oil is greater in the near-wall area. In contrast, when the particle concentration $(0.65,0.97$, and $3.00 \mathrm{ppm})$ is smaller, the variation range of the normal pulsation intensity in the central region is greater. This is because increasing the particle concentration makes the wall surface prone to the rough surface, and this effect enhances the turbulent burst behaviour near the wall, thus leading to a significant increase in the normal pulsation intensity near the wall (Li et al., 2012).

Figure 3(b) shows the distribution of the normal pulsation intensity of the oil under the diameter of $15 \mu \mathrm{m}$ particle size along the normal direction. The influence of different particle concentrations is similar to that of $5 \mu \mathrm{m}$. Figures 3(c) and (d) show that as the particle size increases, the amplitude of the normal pulsation intensity increases slightly, which indicates that the particle size influences the normal pulsation intensity of the oil. 

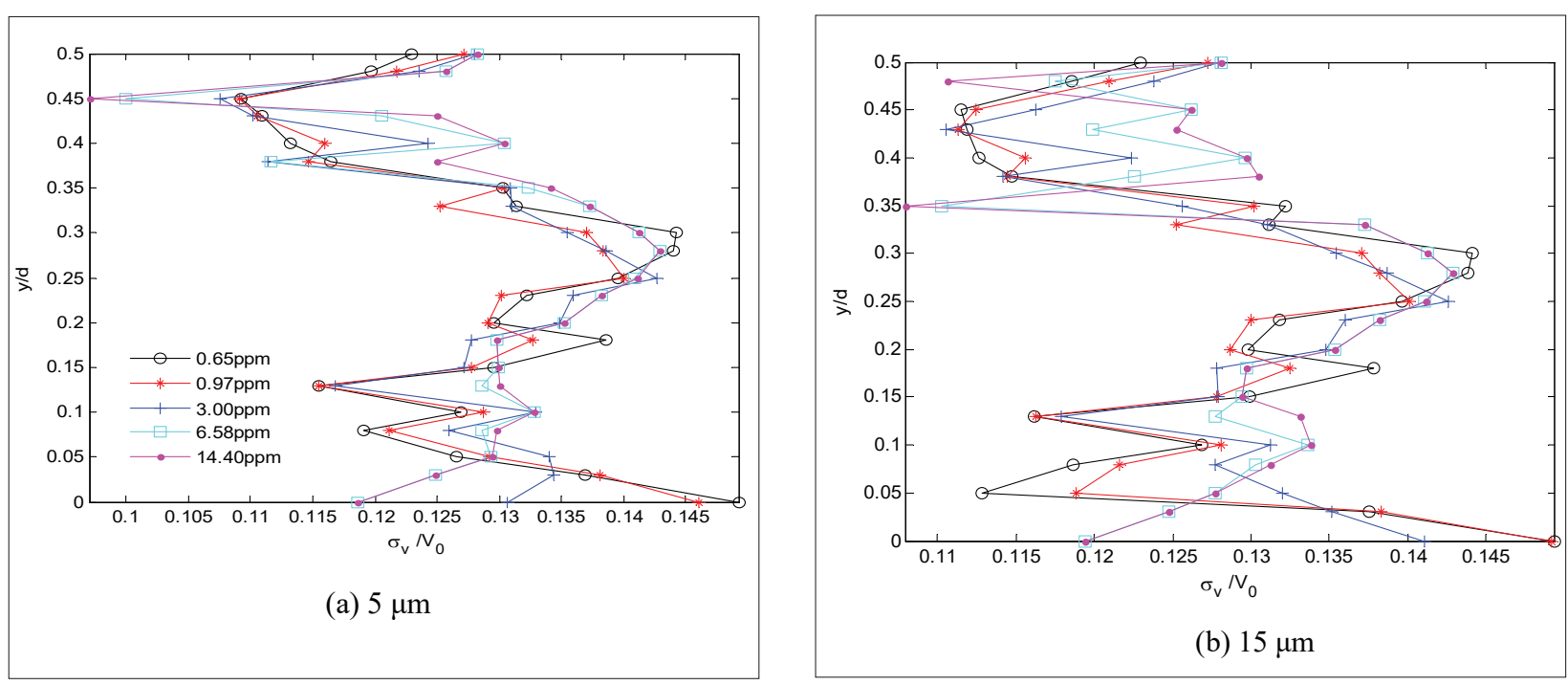

(b) $15 \mu \mathrm{m}$
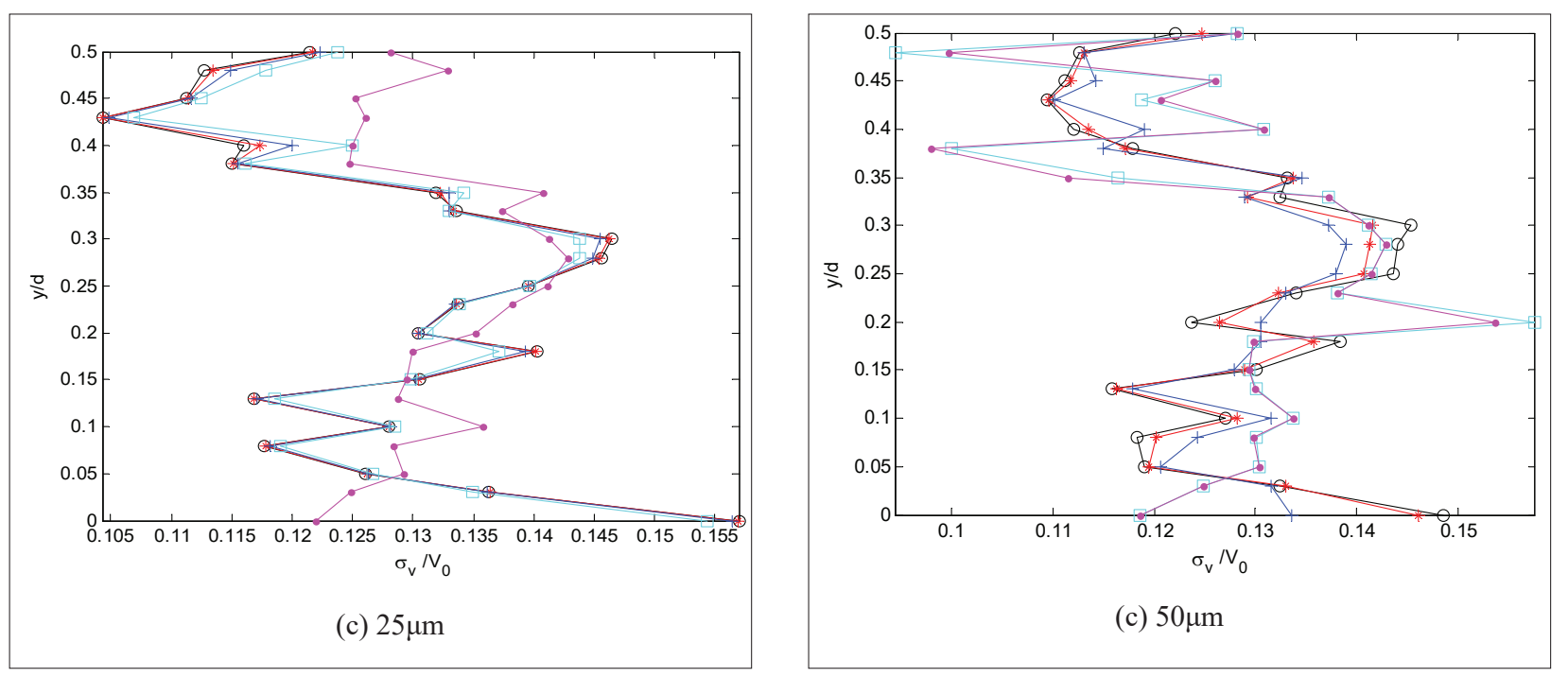

Figure 3: The distribution of the normal pulse velocity along the normal direction

Figure 3(c) shows that due to a decrease in the viscous shear force of $25 \mu \mathrm{m}$ particles in the near-wall region, the distribution of the normal pulsation intensity for different particle concentrations increases with increasing particle concentration. However, in the central region, due to the central mainstream, the retardation role of the particles increases, so the normal pulsation intensity decreases as the particle concentration increases.

\section{Time-frequency curves of transient velocity}

The distribution of pulsation intensity in the flow direction and normal direction shows that the pulsation intensity in the flow direction and normal direction increases simultaneously with the increase of particle size. The particle size of $25 \mu \mathrm{m}$ has a more obvious effect on them. In order to characterize the development of the pulsation characteristics of the oil flow field containing particles in the pipeline with time, according to the transient velocity vector of the PIV test of the flow field in the pipeline, the oil sample with the particle size of $25 \mu \mathrm{m}$ in the oil is taken as an example. The pulsation frequency of the flow field is set to $0.12 \mathrm{~Hz}$ and a point $(0.9,0.25)$ near the centre of the test field is randomly selected. Timefrequency analysis of the instantaneous flow velocity and normal velocity at different particle concentrations of 
$0.65 \mathrm{ppm}, 0.97 \mathrm{ppm}, 3.00 \mathrm{ppm}, 6.58 \mathrm{ppm}$, and $14.40 \mathrm{ppm}$ are shown in Figures 4-8, respectively. Figures 4-8, (a) shows the time curve of the instantaneous flow velocity of the oil, (b) shows the time curve of the instantaneous normal velocity of the oil, (c) presents the spectrum curve of the instantaneous flow velocity of the oil, and (d) presents the spectral curve of the instantaneous normal velocity of the oil.

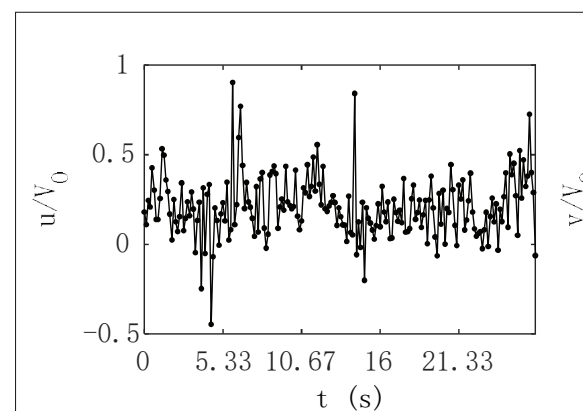

(a)

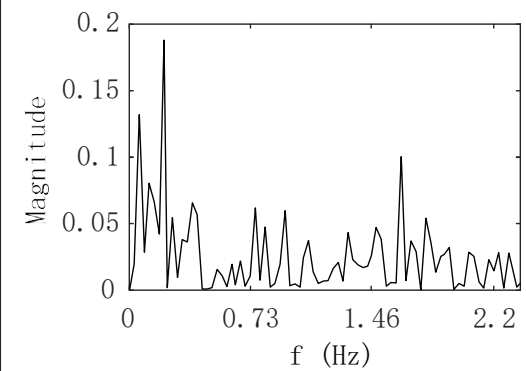

(c)

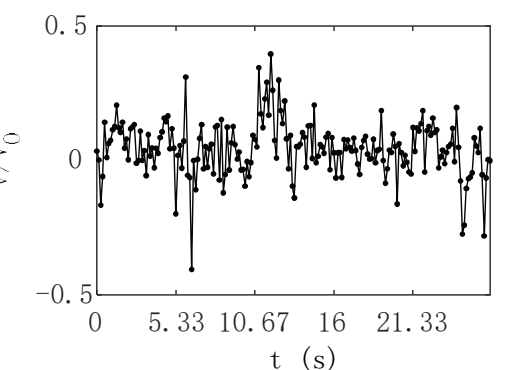

(b)

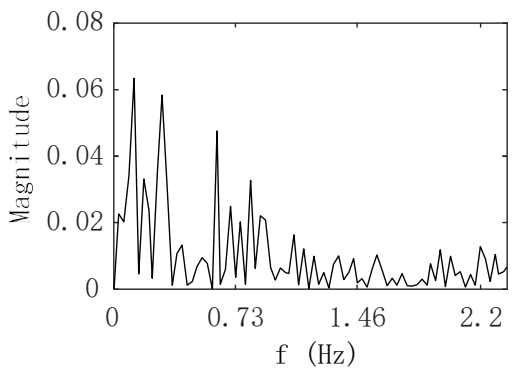

(d)

Figure 4: The time-frequency variation in the transient velocity of oil when the concentration is $0.65 \mathrm{ppm}$

Figure 4 shows the variation in the time-frequency curves of the instantaneous flow velocity and the instantaneous normal velocity when the particle concentration is $0.65 \mathrm{ppm}$. Figure 4(a) presents the change in the instantaneous flow velocity over time. The instantaneous flow velocity fluctuates at approximately 0.2099 under the action of a flow field pulsation base frequency of $0.12 \mathrm{~Hz}$ [as shown in Figure 4(c)], and the flow velocity appears to have a reflow phenomenon (having a negative velocity) in the first cycle; however, the distribution of the flow velocity tends to be gentle after this cycle. Due to the small particle concentration, the viscous shear force has little effect on the oil, where this force made the pulsation degree increase from -0.4474 to 0.9041 , namely, a change of 1.3515. In addition, Figure 4(c) shows that the flow velocity pulsation frequency is mainly $0.21 \mathrm{~Hz}$, approximately two times the base frequency.

Figure 4(b) presents the change in the instantaneous normal velocity with time, and this figure shows that the instantaneous normal velocity at this point fluctuated around 0.0436 , indicating that the velocity distribution in the central region is relatively balanced in the normal direction. The change in the amplitude of the normal velocity is relatively flat, from 0.4058 to 0.3965 , with a range of 0.8023 . Figure 4(d) shows that the normal velocity pulsation frequency of the oil mainly includes the base frequency of $0.12 \mathrm{~Hz}$, and $0.21 \mathrm{~Hz}$, approximately two times of the base frequency.

The time-frequency variations in the instantaneous flow velocity and normal velocity when the particle concentration is $0.97 \mathrm{ppm}$ are shown in Figure 5. The instantaneous flow velocity changes over time, as shown in Figure 5(a), and the instantaneous flow velocity of the point fluctuation is near the value of 0.1683 . Under the action of the flow pulsation base frequency of $0.12 \mathrm{~Hz}$ [as shown in Figure 5(c)], the flow velocity appears to reflow in the first two cycles. Compared with Figure 4(a), as the particle concentration increases and the 
viscous shear force increases, the flow velocity pulsation intensity decreases from -0.3985 to 0.8943 ; namely, the variation range is 1.2928 . In addition, the flow velocity pulsation frequency is $0.18 \mathrm{~Hz}$, which is 1.5 times the base frequency (shown in Figure 5(c)].

Figure 5(b) shows the curve of the change in the instantaneous normal velocity over time. The instantaneous normal velocity of the point fluctuates near the value of 0.0299 , which shows that the normal velocity distribution in the central region is balanced concerning that of Figure 4(b). However, the magnitude of the change in the normal velocity is relatively large, ranging from -0.4759 to 0.4649 , with a variation of 0.9408 . Figure $5(\mathrm{~d})$ shows that the normal velocity pulsation frequency mainly includes the subharmonic frequency of $0.09 \mathrm{~Hz}$, and $0.21 \mathrm{~Hz}$, approximately twice the base frequency.

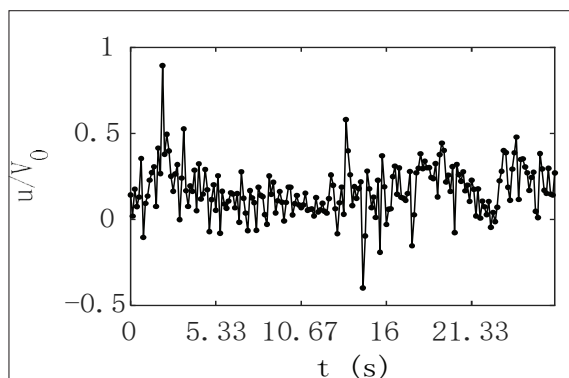

(a)

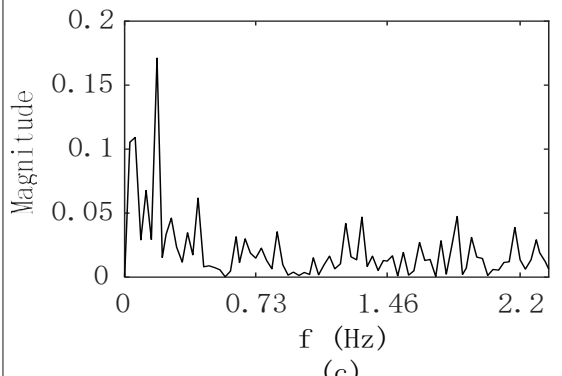

(c)

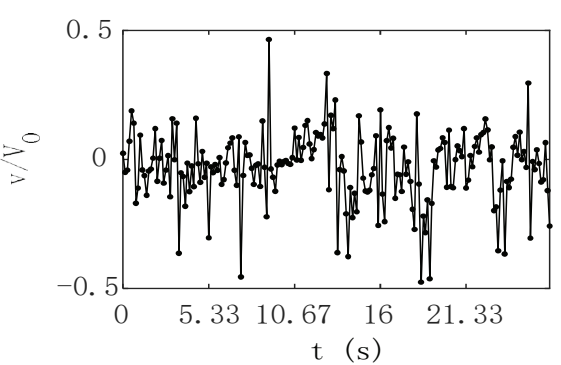

(b)

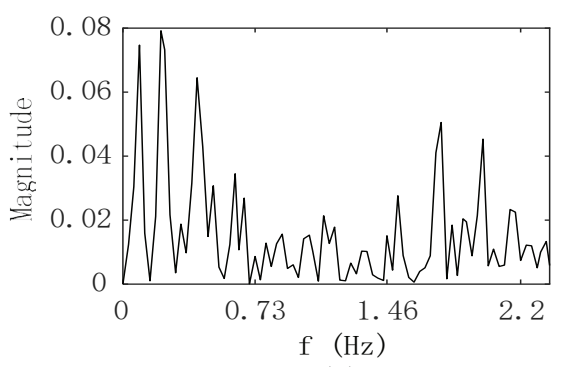

(d)

Figure 5: The time-frequency variation in the transient velocity of oil when the concentration is $0.97 \mathrm{ppm}$

Figure 6 shows the time-frequency variations in the instantaneous flow velocity and normal velocity when the particle concentration is $3.00 \mathrm{ppm}$. The instantaneous flow velocity changes over time, as shown in Figure 6(a), and the transient flow velocity of the point fluctuates near a value of 0.1635 . Under the action of the flow field base pulsation frequency of $0.12 \mathrm{~Hz}$ [as shown in Figure 6(c)], the flow velocity amplitude in the first period has a large fluctuation phenomenon, and the reflow phenomenon occurs in the third cycle. As the particle concentration increases and the viscous shear force of the oil increases, the flow velocity pulsation degree of the oil shown in Figure 6(a) relative to that of Figure 4(a) and Figure 5(a) decreases from -0.1292 to 0.8407 , with a varied range of 0.9699. In addition, Figure 6(c) shows that the flow velocity pulsation frequency is mainly $0.09 \mathrm{~Hz}$, which is a subharmonic frequency of the base frequency.
In Figure 6(b), the instantaneous normal velocity at a randomly selected point $(0.9,0.25)$ near the centre of the test field fluctuates around -0.0387 over time, which indicates that the velocity distribution in the central region has a negative trend in the normal direction relative to that of Figure 4(b) and Figure 5(b). However, the magnitude of the change in the normal velocity is relatively small, from -0.3491 to 0.3944 , with a varied range of 0.7435 . Figure 6(d) shows that the normal velocity pulsation frequency is $0.06 \mathrm{~Hz}$, which is a subharmonic frequency of the base frequency.

Figure 7 shows the time-frequency variations in the instantaneous flow velocity and normal velocity when the particle concentration is $6.58 \mathrm{ppm}$. The instantaneous flow velocity of the point shown in Figure 7(a) fluctuates near 0.161 over time. Under the action of 


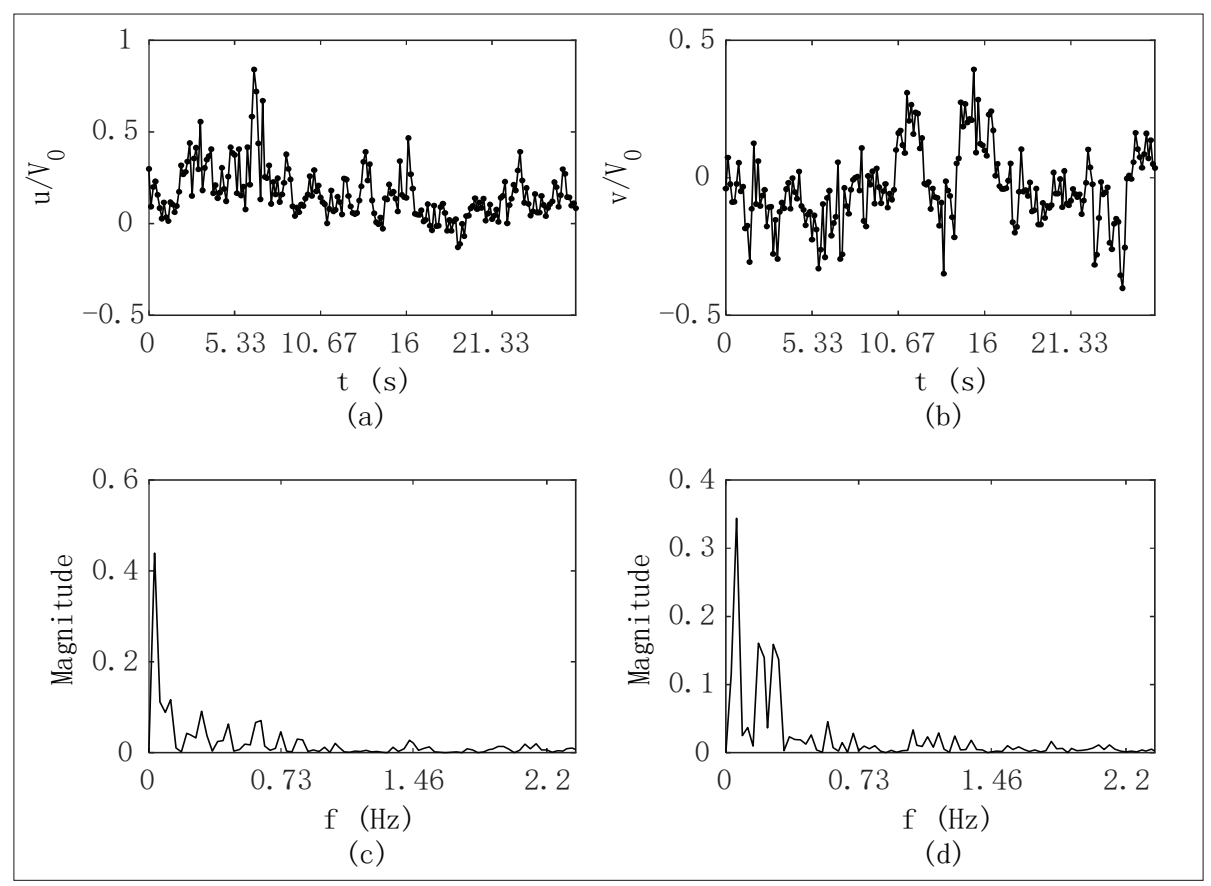

Figure 6: The time-frequency variation in the transient velocity of oil when the concentration is $3.00 \mathrm{ppm}$

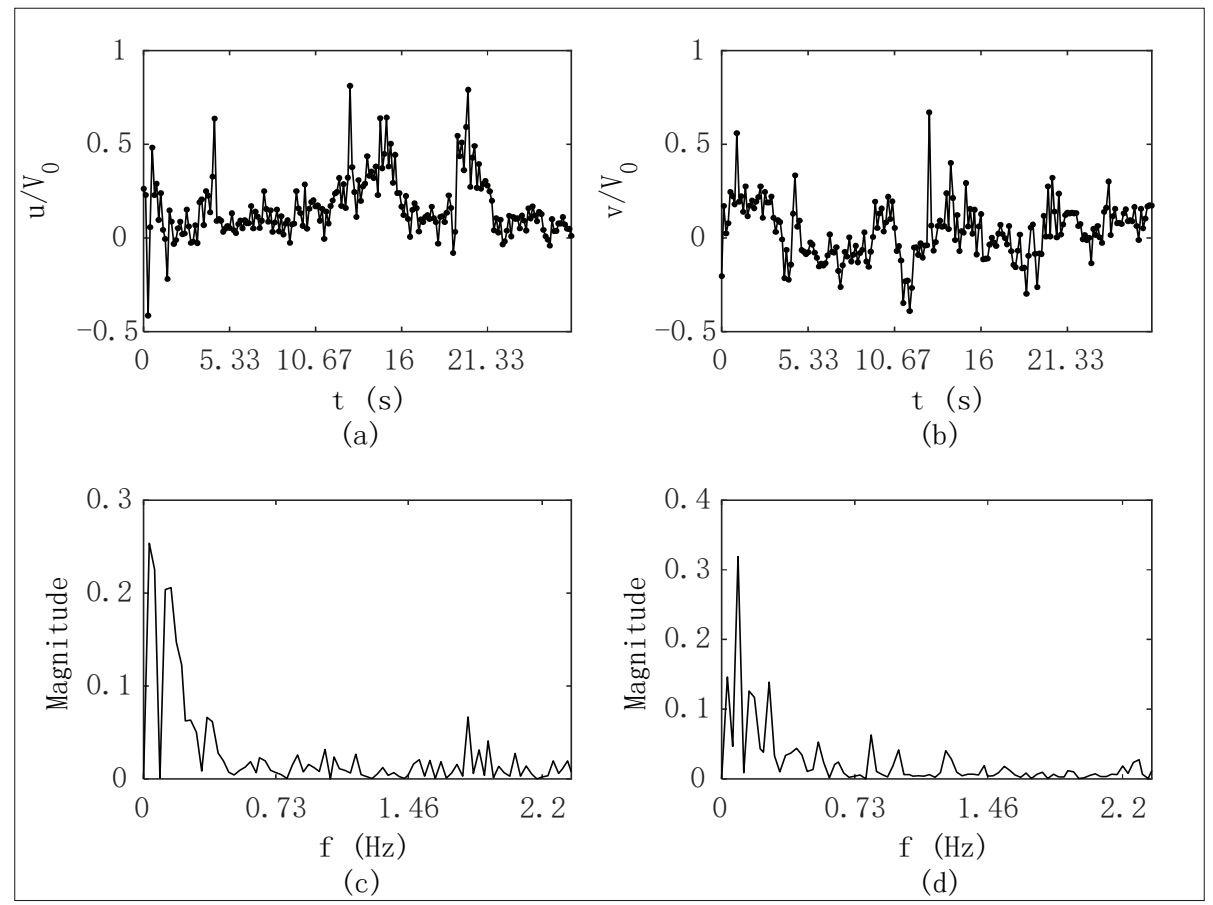

Figure 7: The time-frequency variation in the transient velocity of oil when the concentration is $6.58 \mathrm{ppm}$ 
the flow field pulsation frequency of $0.12 \mathrm{~Hz}$ [as shown in Figure 7(c)], the amplitude of the flow velocity in the latter two periods has a large fluctuation phenomenon. As the particle concentration increases and the viscous shear force increases, the flow velocity pulsation degree is reduced from 0.0807 to 0.8113 relative to that of Figures 4-6(a), with a varied range of 0.8920 . In addition, Figure 7 (c) shows that the flow velocity pulsation frequency is mainly a subharmonic frequency of $0.03 \mathrm{~Hz}$ and a base frequency of $0.12 \mathrm{~Hz}$.
In Figure 7(b), the instantaneous normal velocity of the point fluctuates near 0.032 over time, which shows that in the central region, the normal upward velocity distribution has a positive trend relative to the trend of Figure 4-6 (b). However, the magnitude of the change in the normal velocity is relatively higher from -0.3905 to 0.6698 , with a range of 1.0603. In addition, Figure 7(d) shows that the normal velocity pulsation frequency is mainly the subharmonic frequency of $0.09 \mathrm{~Hz}$ and is approximately twice the frequency of $0.26 \mathrm{~Hz}$ of the base frequency.

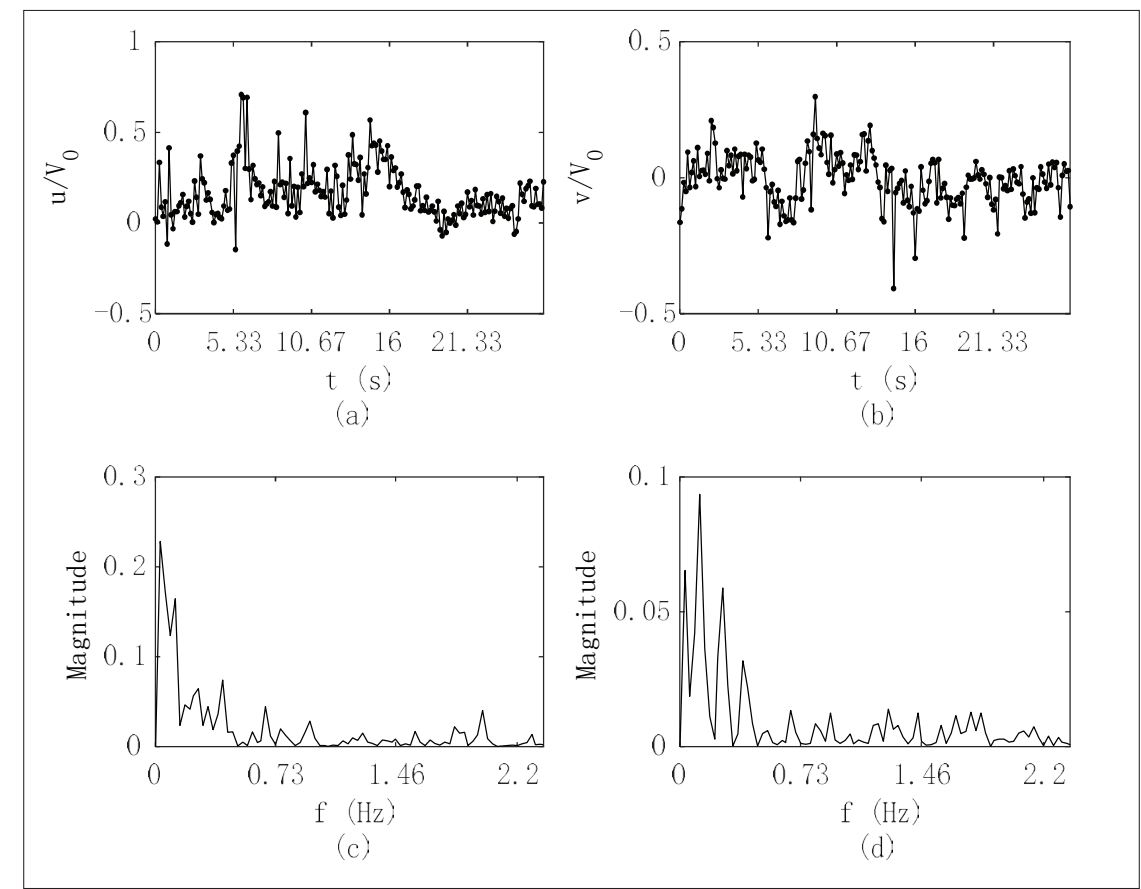

Figure 8: The time-frequency variation in the transient velocity of oil when the concentration is $14.40 \mathrm{ppm}$

Table 1: Fluctuation frequency of the transient velocity in the flow field

\begin{tabular}{lcccccccc}
\hline Velocity & $\begin{array}{c}\text { Concentration } \\
(\mathrm{ppm})\end{array}$ & Subharmonic & $\begin{array}{c}\text { Base } \\
\text { frequency }\end{array}$ & $\begin{array}{c}\text { Second-time } \\
\text { frequency }\end{array}$ & $\begin{array}{c}\text { Third-time } \\
\text { frequency }\end{array}$ & $\begin{array}{c}\text { Fourth-time } \\
\text { frequency }\end{array}$ & $\begin{array}{c}\text { Fifth-time } \\
\text { frequency }\end{array}$ & $\begin{array}{c}\text { Other times } \\
\text { frequency }\end{array}$ \\
\hline \multirow{3}{*}{ Flow } & 0.65 & 0.06 & 0.12 & 0.21 & 0.41 & & & 0.71 \\
velocity & 0.97 & 0.06 & 0.12 & 0.18 & & 0.46 & 0.85 \\
& 3.00 & 0.03 & 0.12 & 0.29 & 0.44 & & 0.62 & 0.73 \\
& 6.58 & 0.03 & 0.12 & 0.26 & 0.35 & & 0.64 & 0.85 \\
Normal & 14.40 & 0.03 & 0.12 & 0.26 & 0.41 & & 0.67 & 0.94 \\
velocity & 0.65 & 0.03 & 0.12 & 0.29 & 0.41 & 0.53 & 0.62 & 0.82 \\
& 0.97 & 0.09 & & 0.21 & 0.41 & 0.50 & 0.62 & 0.88 \\
& 3.00 & 0.06 & 0.18 & 0.26 & 0.47 & 0.56 & & 0.70 \\
& 6.58 & 0.09 & 0.18 & 0.26 & 0.41 & 0.53 & & 0.82 \\
\hline
\end{tabular}


Figure 8 shows the time-frequency variations in the instantaneous flow velocity and normal velocity when the particle concentration is $14.40 \mathrm{ppm}$. As shown in Figure 8(a), the instantaneous flow velocity at this point fluctuates around 0.166 over time. Under the action of the flow field pulsation frequency of $0.12 \mathrm{~Hz}$ [as shown in Figure 8(c)], the flow velocity amplitude appears to exhibit large fluctuations in the first two cycles. As the particle concentration increases and the viscous shear force increases, the flow velocity pulsation degree decreases concerning that of Figures 4-7(a), from -0.1459 to 0.6922 with a varied range of 0.8381 .
In addition, Figure 8(c) shows that the flow pulsation frequency is mainly a subharmonic frequency of $0.03 \mathrm{~Hz}$ and a base frequency of $0.12 \mathrm{~Hz}$.

As shown in Figure 8(b), the instantaneous normal velocity at this point fluctuates around -0.008 , which indicates that the normal velocity distribution in the central region is relatively balanced concerning that of Figure 4-7(b). However, the magnitude of the change in the normal velocity is relatively small, ranging from -0.4071 to 0.2982 with a varied range of 0.7053 . In addition, Figure $8(\mathrm{~d})$ shows that the normal velocity

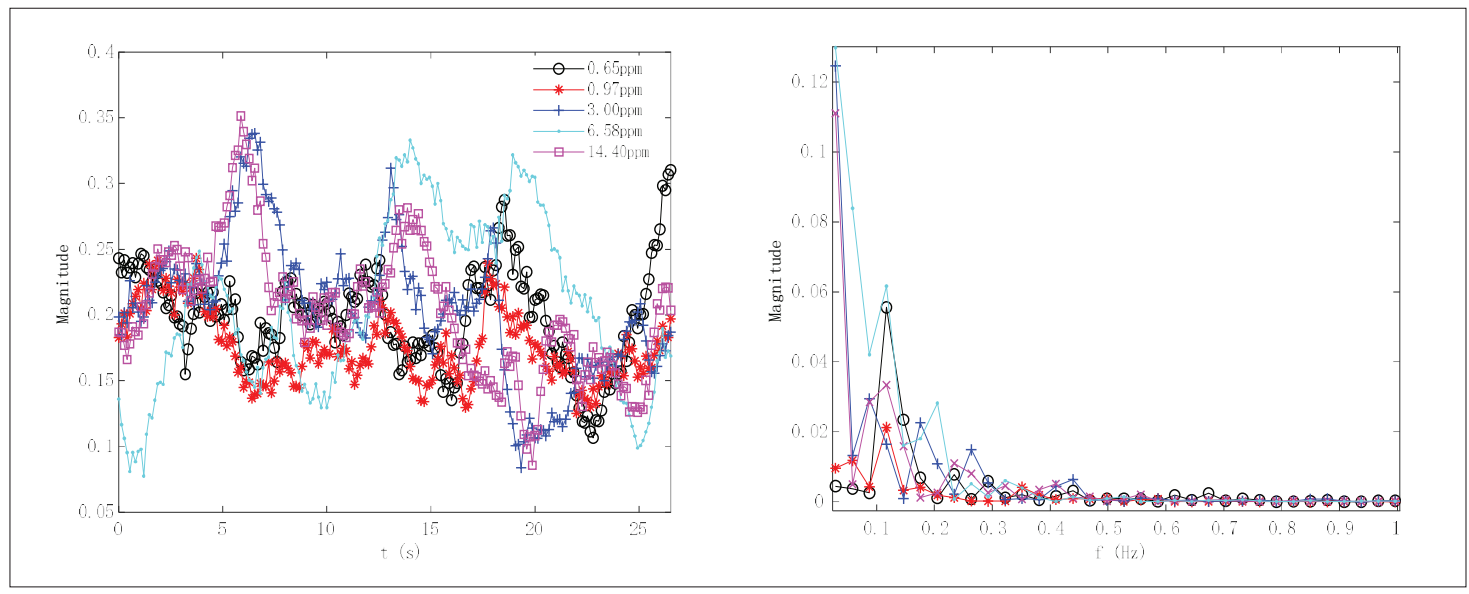

(a) The time-domain distribution of the average flow velocity (b) The frequency-domain distribution of the average flow velocity

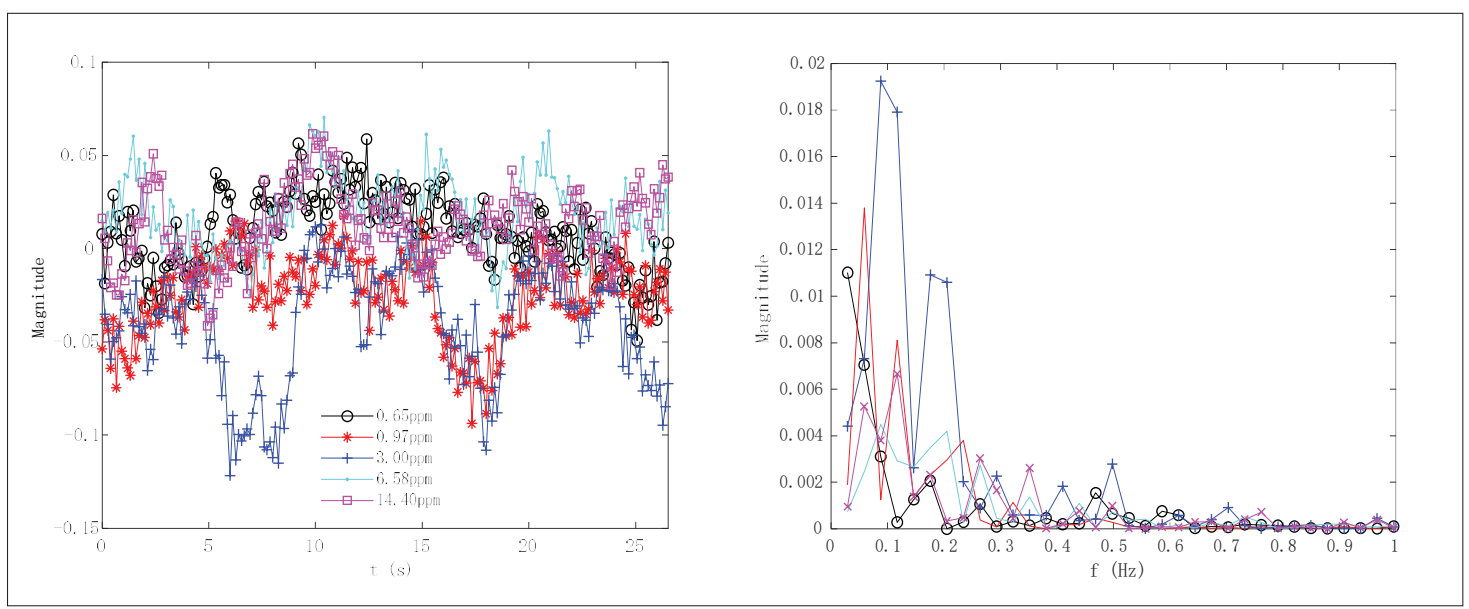

(c) The time-domain distribution of the normal average velocity (d) The frequency-domain distribution of the normal average velocity

Figure 9: The time-frequency curves of the mean velocity of oil 
pulsation frequency is mainly the subharmonic frequency of $0.03 \mathrm{~Hz}$ and is approximately twice the frequency of $0.26 \mathrm{~Hz}$ of the base frequency.

The above results show that with increasing particle concentration in oil, the mean value of the magnitude of the instantaneous flow velocity pulsation is gradually reduced, and the varying amplitude simultaneously decreases. On the other hand, the changes in the mean and variance amplitude of the instantaneous normal velocity pulsation are not obvious due to the influence of the flow field structure.

Table 1 shows the main frequencies of the instantaneous flow velocity and normal velocity for different particle concentrations. The base frequency of $0.12 \mathrm{~Hz}$ is reflected in the frequency domain of the flow velocity. As the particle concentration increases, the main frequency of the flow velocity decreases from twice the base frequency to the subharmonic frequency, which shows an attenuation trend. Similarly, with increasing particle concentration, the main frequency of the normal velocity of oil decreases from the base frequency to the subharmonic frequency, which also shows an attenuation trend.

\section{The speed-frequency curve of average velocity}

The instantaneous velocity vectors for different particle concentrations obtained by the PIV test are averaged in the flow direction and the normal direction to obtain the corresponding time-frequency curves of the average flow velocity and normal average velocity of oil in the time of the PIV test, as shown in Figure 9.

Figure 9(a) shows the time-domain distribution of the average flow velocity under different particle concentrations; it is obvious that the distribution of the average flow velocity is periodic. In addition, the base frequency of the flow field is $0.12 \mathrm{~Hz}$, as shown in Figure 9(b). With increasing particle concentration, the change in the average flow velocity is non-unidirectional, but the overall trend is that the average velocity of the oil gradually increases. The reason is that due to the increase in the particle concentration, the particle inertia increases the average velocity in the near-wall region, so the thickness of the turbulent viscous bottom layer decreases and the flow velocity gradient increases (Li et al., 2012), which lead to an increase in the flow pulsation peak value.

The time-domain distribution of the average normal velocity for different particle concentrations is shown in Figure 9(c). As the particle concentration increases, the change in the average normal velocity decreases up to a particle concentration of $3.00 \mathrm{ppm}$, while this change increases when the particle concentration exceeds 3.00 ppm. This is because when the particle concentration reaches a certain level $(3.00 \mathrm{ppm})$ in the horizontal pipeline, the sedimentation of the particles causes the wall surface to form a rough wall surface, which enhances the release of the oil with a sudden turbulence behaviour in the near-wall region and results in increasing the normal average velocity. Figure 9(d) shows that the main frequency of the normal average velocity is concentrated in a subharmonic frequency of $0.09 \mathrm{~Hz}$ and a base frequency of $0.12 \mathrm{~Hz}$, which is consistent with the main frequency of the transient normal velocity

\section{CONCLUSIONS}

Faults of power transformer such as loose cores and coil deformation can be detected by transformer pulsation. Compared to detecting pulsation of the transformer body, detecting pulsation of the transformer oil is more accurate. However, the inevitable presence of metallic and nonmetallic particles in transformer oil, their particle size, and concentration also plays a non-negligible influence on the pulsation of the oil. Therefore, it is important to research the influence of factors such as particle size and concentration on the pulsation characteristics of the oil for the safe operation of the transformer. According to the ISO 4406 standard for oil contamination, oil samples containing $\mathrm{Cu}, \mathrm{Fe}$, and $\mathrm{SiO}_{2}$ powders with particle sizes of $5,15,25$ and $50 \mu \mathrm{m}$ and concentrations of $6.58,4.57$, $3.00,0.971$, and $0.648 \mathrm{ppm}$ were prepared and tested in an experimental test setup using the 2DPIV measurement technique for different oil samples in a square tube, to obtain the transient velocity vectors of the oil flow field containing particles of different particle sizes and concentrations. The distribution of the oil flow direction and normal pulsation intensity, as well as the transient and average velocities, were analysed. It was found that the distribution of the oil flow pulsation intensity along the normal direction changes slowly in the central region, while the change in the near-wall region is steep, and this trend becomes more obvious with increasing particle concentration. In addition, the flow direction pulsation intensity of oil decreases with increasing particle concentration, and the difference gradually decreases. The distribution of the normal pulsation strength of oil in the normal direction for different particle sizes is roughly of the shape of a ' $W$ '. The amplitude of the normal pulsation intensity fluctuates greatly in the central region and near-wall region. In addition, the influence of particle concentration on the distribution of the normal pulsation intensity is non-unidirectional. With 
increasing particle concentration, the mean value of the fluctuation amplitude of the instantaneous flow velocity gradually decreases, and the varying amplitude decreases simultaneously. However, the fluctuation amplitude of the instantaneous normal velocity is not obvious. With increasing particle concentration, the main frequencies of the flow direction and normal direction velocities show a decreasing trend. The distribution periodicity of the average flow velocity of oil is more obvious. As the particle concentration increases, the average flow velocity gradually increases. When the particle concentration is below $3.00 \mathrm{ppm}$, the average normal velocity decreases with increasing particle concentration, while when the particle concentration exceeds $3.00 \mathrm{ppm}$, the average normal velocity of the oil shows an increasing trend. It can be seen that the influence of particles on the transformer oil pulsation is large. For the transformer pulsation test it is necessary to consider the influence of these factors in order to accurately obtain the operating state of the transformer for safe operation of the transformer.

\section{Acknowledgements}

This study was funded by Local Science and Technology Development Fund projects guided by the central government (206Z5201G), Chongqing Basic Research and Frontier Exploration Project (cstc2018jcyjAX0121), and Fundamental Research Funds for the Central Universities (3142019001, 3142019055).

\section{REFERENCES}

Abiev R.S. \& Galushko A.S. (2013). Hydrodynamics of pulsating flow type apparatus: Simulation and experiments. Chemical Engineering Journal 229: 285-295.

DOI: https://doi.org/10.1016/j.cej.2013.05.105

Bagheri M., Nezhivenko S., Naderi M.S. \& Zollanvari A. (2018). A new vibration analysis approach for transformer fault prognosis over cloud environment. International Journal of Electrical Power and Energy Systems 100: 103-116.

DOI: https://doi.org/10.1016/j.ijepes.2018.02.026

Borges G.R., Farias G.B., Braz T.M., Santos L.M., Amaral M.J., Fortuny M., Franceschi E., Dariva C. \& Santos A.F. (2015). Use of near-infrared for evaluation of droplet size distribution and water content in water-in-crude oil emulsions in pressurized pipeline. Fuel 147: 43-52. DOI: https://doi.org/10.1016/j.fuel.2015.01.053

Eschmann G., Kuntze A., Uffrecht W., Kaiser E. \& Odenbach S. (2015). Experimental and numerical investigation of heat transfer coefficients in gaseous impinging jets - First test of a recent sensor concept for steady and unsteady flow. International Journal of Thermal Sciences 96: 290-304. DOI:https://doi.org/10.1016/j.ijthermalsci.2015.03.016

Ghadi S., Esmailpour K., Hosseinalipour S.M. \& Mujumdar A. (2016). Experimental study of formation and development of coherent vortical structures in pulsed turbulent impinging jet. Experimental Thermal and Fluid Science 74: 382-389. DOI:https://doi.org/10.1016/j.expthermflusci.2015.12.007

Hsu C.M., Huang R.F. \& Loretero M.E. (2014). Unsteady flow motions of an oscillating jet in crossflow. Experimental Thermal and Fluid Science 55: 77-85.

DOI:https://doi.org/10.1016/j.expthermflusci.2014.02.016

Li J., Wang H., Liu Z., Chen S. \& Zheng C. (2012). An experimental study on turbulence modification in the nearwall boundary layer of a dilute gas-particle channel flow. Experiments in Fluids 53(5): 1385-1403.

DOI: https://doi.org/10.1007/s00348-012-1364-7

Ling L. \& Zhong X. (1999). Effect of particle concentration and size on distribution of velocity for the gas-particle two-phase flow in a vertical square duct. Journal of Hydrodynamics 14(2): 154-161.

Papadopoulos P.K. \& Vouros A.P. (2016). Pulsating turbulent pipe flow in the current dominated regime at high and veryhigh frequencies. International Journal of Heat and Fluid Flow 58: 54-67. DOI:https://doi.org/10.1016/j.ijheatfluidflow.2015.12.007

Tian J., Yuan C., Yang L., Wu C., Liu G. \& Yang Z. (2016). The vibration analysis model of pipeline under the action of gas pressure pulsation coupling. Engineering Failure Analysis 66: 328-340.

DOI:https://doi.org/10.1016/j.engfailanal.2016.05.017

Wang G., Evans G.M. \& Jameson G.J. (2016). Bubble-particle detachment in a turbulent vortex I: Experimental. Minerals Engineering 92: 196-207. DOI: https://doi.org/10.1016/j.mineng.2016.03.011

Yan B.H., Gu H.Y. \& Yu L. (2012). Numerical simulation of large scale vortex structure and flow pulsation in rectangular channels. Progress in Nuclear Energy 54(1): 29-35. DOI: https://doi.org/10.1016/j.pnucene.2011.09.006

Yang B., Zhang W. \& Zhang Y. (2013). Experiment study of the wind fluctuation's effect on the sand concentration in unsteady wind-sand flow. Journal of Experiments in Fluid Mechanics 27(3): 47-50.

Yuan H., Tan S., Wen J. \& Zhuang N. (2016). Heat transfer of pulsating laminar flow in pipes with wall thermal inertia. International Journal of Thermal Sciences 99: 152-160. DOI:https://doi.org/10.1016/j.ijthermalsci.2015.08.014

Zeng L., Yu Z., Zhang H., Zhang X. \& Chen H. (2018). A high sensitive multi-parameter micro sensor for the detection of multi-contamination in hydraulic oil. Sensors and Actuators: A. Physical 282: 197-205.

DOI: https://doi.org/10.1016/j.sna.2018.09.023

Zhao H., Wei A., Luo S. \& Fan J. (2016). Velocity fluctuations in the near-wall region of the turbulent channel flow. Journal of Engineering Thermal Physics 37(3): 551-555. 